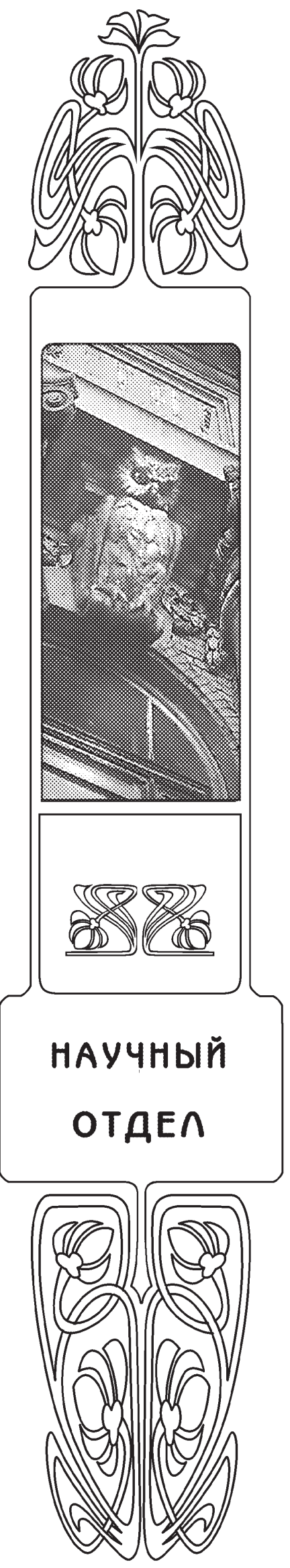

\title{
ИНФОРМАТИКА
}

\section{Hybrid Automation Extended Model}

\section{K. Andreichenko, K. P. Andreichenko, I. A. Batraeva}

Dmitry K. Andreichenko, https://orcid.org/0000-0003-0525-984X, Saratov State University, 83 Astrakhanskaya St., 410012 Saratov, Russia, andreichenkodk@gmail.com

Konstantin P. Andreichenko, https://orcid.org/0000-0002-8729-4317, Yuri Gagarin State Technical University of Saratov, 77 Politechnicheskaya St., 410054 Saratov, Russia, kp_andreichenko@ renet.ru

Inna A. Batraeva, https://orcid.org/0000-0002-6539-8473, Saratov State University, 83 Astrakhanskaya St., 410012 Saratov, Russia, batraevaia@info.sgu.ru

An extended model of hybrid automata for dynamic systems is considered, where, along with a discrete control subsystem and control objects with lumped parameters, there are control objects with distributed parameters (linear and stationary from the point of view of automatic control theory). The possibility of software implementation of an extended model of hybrid automata on embedded computing systems is shown.

Keywords: hybrid automata, hybrid dynamical systems, embedded computing systems.

Received: 21.10.2018 / Accepted: 22.12.2018

Published online: 28.02.2019

DOI: https://doi.org/10.18500/1816-9791-2019-19-1-94-104

\section{INTRODUCTION. MODEL OF THE HYBRID AUTOMATA}

A hybrid automaton is a mathematical model of a hybrid system, where the switchings of a discrete control subsystem are modeled based on automata theory, and the motion of control objects with lumped parameters is based on Cauchy problems for ordinary differential equations [1-7].

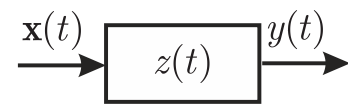

Fig. 1. Structural scheme

We consider the input-output model of a dynamic system with time $t \in \mathbb{R}$, a piecewise continuous input function $\mathbf{x}(t)$, $\mathbf{x}: \mathbb{R} \rightarrow X \subset \mathbb{R}^{N_{x}}$, an output function $y(t), y: \mathbb{R} \rightarrow Y$ and a function $z(t), z: \mathbb{R} \rightarrow Z$, characterizing the time variation of the state of a dynamic system (Fig. 1). The system contains discrete control elements and control objects with lumped parameters interacting with each other. 
Respectively

$$
\begin{gathered}
Z=S \times Z_{c}, \quad|S|<\infty, \quad Z_{c} \in \mathbb{R}^{N_{z c}}, \quad z=\left(s, \mathbf{z}_{c}\right), \quad s \in S, \quad \mathbf{z}_{c} \in Z_{c}, \\
Y=Y_{d} \times Y_{c}, \quad\left|Y_{d}\right|<\infty, \quad Y_{c} \in \mathbb{R}^{N_{y}}, \quad y=\left(y_{d}, \mathbf{y}_{c}\right), \quad y_{d} \in Y_{d}, \quad \mathbf{y}_{c} \in Y_{c} .
\end{gathered}
$$

Here $S$ is the finite set of states of the discrete subsystem, $Y_{d}$ is the finite set of values of the discrete outputs, $Z_{c}$ is the set of states of the continuous subsystem, $Y_{c}$ is the set of values of the continuous outputs. The time evolution of a discrete control subsystem is characterized by a sequence of pairs of time intervals and the values of the states of the discrete subsystem that remain on them $\left\{\left(\left[0, t_{1}\right], s_{0}\right),\left(\left[t_{1}, t_{2}\right], s_{1}\right), \ldots,\left(\left[t_{j}, t_{j+1}\right], s_{j}\right), \ldots\right\}$. Therefore

$$
T=\left\{t_{1}, t_{2}, \ldots, t_{j}, t_{j+1}, \ldots\right\}
$$

is the sequence of switching times. The classic model of the hybrid automaton [1-7] is a tuple

$$
A_{H}=\left\langle Z, X, Y, z_{0}, R, f_{p}, \mathbf{f}_{r}, \mathbf{f}_{c}, f_{o}\right\rangle
$$

where $z_{0}=\left(s_{0}, \mathbf{z}_{c_{0}}\right) \in Z=S \times Z_{c}$ is the initial state of the system; $R=\left\{\left(s_{k}, s_{j}\right)\right\} \subset S \times S$ is the set of arcs of the oriented graph $(S, R)$ which determines the possible transitions between the states of the discrete subsystem during its switching; $f_{p}: \mathbb{R} \times X \times R \times Z_{c} \rightarrow\{0,1\}$ is a predicate function that allows or prohibits switching of a discrete subsystem. For the sequence of switching times (2) is true

$$
f_{p}\left(t_{j},\left.\mathbf{x}\right|_{t_{j}-0},\left(s_{j-1}, s_{j}\right),\left.\mathbf{z}_{c}\right|_{t_{j}-0}\right)=1, \quad t_{j} \in T, \quad j=1,2,3, \ldots
$$

Function $\mathbf{f}_{r}: \mathbb{R} \times X \times R \times Z_{c} \rightarrow Z_{c}$ is a function of updating initial conditions at discrete switching

$$
\left.\mathbf{z}_{c}\right|_{t_{j}+0}=\mathbf{f}_{r}\left(t_{j},\left.\mathbf{x}\right|_{t_{j}-0},\left(s_{j-1}, s_{j}\right),\left.\mathbf{z}_{c}\right|_{t_{j}-0}\right), \quad j=1,2,3, \ldots
$$

Function $\mathbf{f}_{c}: \mathbb{R} \times X \times S \times Z_{c} \rightarrow Z_{c}$ is a derivative function defining the right-hand sides of ordinary differential equations, which model the dynamics of a continuous subsystem over time intervals corresponding to persisting states of the discrete subsystem

$$
\begin{gathered}
\dot{\mathbf{z}}_{c}=\mathbf{f}_{c}\left(t, \mathbf{x}, s_{0}, \mathbf{z}_{c}\right), \quad \mathbf{z}_{c}(0)=\mathbf{z}_{c_{0}}, \quad t \in\left[0, t_{1}\right], \quad(\dot{)}=d() / d t, \\
\dot{\mathbf{z}}_{c}=\mathbf{f}_{c}\left(t, \mathbf{x}, s_{j}, \mathbf{z}_{c}\right), \quad \mathbf{z}_{c}\left(t_{j}\right)=\left.\mathbf{z}_{c}\right|_{t_{j}+0}, \quad t \in\left[t_{j}, t_{j+1}\right], \quad j=1,2,3, \ldots
\end{gathered}
$$

Here $\left.\mathbf{z}_{c}\right|_{t_{j}+0}$ and $\left.\mathbf{z}_{c}\right|_{t_{j}-0}, j=1,2,3, \ldots$ are connected by equation (5). $f_{0}: \mathbb{R} \times X \times S \times Z_{c} \rightarrow Y$ is an output function

$$
y=f_{0}\left(t, \mathbf{x}, s_{j}, \mathbf{z}_{c}\right), \quad j=1,2,3, \ldots
$$

In order for the hybrid automaton to be deterministic, it is required that when $t \geqslant 0$ the predicate function $f_{p}$ can take the value 1 on no more than one arc of the graph $(S, R)$, originating from the current state of the discrete subsystem. The hybrid automaton is effective software implemented on embedded computing systems. It is of interest to include this class of mathematical models of control objects with distributed parameters [8], and, accordingly, initial-boundary problems for partial differential equations, but with the possibility of efficient software implementation on embedded computing systems. 


\section{SPECIFICATION OF THE ORIGINAL MODEL}

Depending on the specific type of predicate $f_{p}$ and function $\mathbf{f}_{r}$, at the moment of time $t_{j} \in T, j=1,2,3, \ldots$ there can be either a single switches of the states of the discrete subsystem or a cascade of switching. However, the switching cascade is undesirable due to energy and structural constraints. We assume that switching cascades are excluded, and in (2)

$$
0<t_{1}<t_{2}<\ldots<t_{j}<t_{j+1}<\ldots
$$

We assume that the initial state of a dynamical system is an equilibrium state, from which the dynamical system is derived by varying the input vector function with time $t \geqslant 0$. Those

$$
\mathbf{x}(t)=\mathbf{x}_{0}=\text { const }, \quad t<0 ; \quad f_{c}\left(t, \mathbf{x}_{0}, s_{0}, \mathbf{z}_{c 0}\right)=0, \quad t \leqslant 0
$$

Formally, the sequence of Cauchy problems (6) is equivalent to one Cauchy problem

$$
\begin{gathered}
\dot{\mathbf{z}}_{c}=\mathbf{f}\left(t, \mathbf{x}, \mathbf{z}_{c}\right), \quad \mathbf{f}\left(t, \mathbf{x}, \mathbf{z}_{c}\right)=\sum_{j=1,2,3, \ldots}\left(\left.\mathbf{z}_{c}\right|_{t_{j}+0}-\left.\mathbf{z}_{c}\right|_{t_{j}-0}\right) \delta\left(t-t_{j}\right)+ \\
+ \begin{cases}\mathbf{f}_{c}\left(t, \mathbf{x}, s_{0}, \mathbf{z}_{c}\right), & t \in\left[0, t_{1}\right], \\
\mathbf{f}_{c}\left(t, \mathbf{x}, s_{j}, \mathbf{z}_{c}\right), & t \in\left[t_{j}, t_{j+1}\right], \quad j=1,2,3, \ldots \\
& \mathbf{z}_{c}(0)=\mathbf{z}_{c_{0}}\end{cases}
\end{gathered}
$$

where $\delta(t)$ is the Dirac impulse function. In (10) $\left.\mathbf{z}_{c}\right|_{t_{j}+0}$ and $\left.\mathbf{z}_{c}\right|_{t_{j}-0}, j=1,2,3, \ldots$ are related by equation (5). If the continuous dynamic subsystem contains some links with constant or variable positive lag periods, and $\tau_{\max }$ is the maximum lag time, the initial conditions (11) take the form

$$
\mathbf{z}_{c}(t)=\mathbf{z}_{c_{0}}, \quad t \in\left[-\tau_{\max }, 0\right] .
$$

The mathematical model of the hybrid automaton (1)-(7) or (1)-(5), (7), (8)-(10), (12) is the most feasible mathematical model in the MATLAB / Simulink / Stateflow dynamic system simulation software. MATLAB/ Simulink standard tools provide the generation of program code for the software implementation of this class of dynamic systems on embedded computing systems. For further, it is advisable to separate linear and nonlinear terms as a function of derivatives

$$
\begin{gathered}
\mathbf{f}_{c}\left(t, \mathbf{x}, s, \mathbf{z}_{c}\right)=B \mathbf{x}+C \mathbf{z}_{c}+\sum_{k=1}^{N_{\tau}} C_{k}^{(\tau)} \mathbf{z}_{c}\left(t-\tau_{k}\right)+\mathbf{f}_{c}^{(1)}\left(t, \mathbf{x}, s, \mathbf{z}_{c}\right), \\
\mathbf{f}_{c}^{(1)}: \mathbb{R} \times X \times S \times Z_{c} \rightarrow Z_{c}, \\
B=\mathrm{const} \in \mathbb{R}^{\left(N_{z c}, N_{x}\right)}, \quad C=\mathrm{const} \in \mathbb{R}^{\left(N_{z c}, N_{z c}\right)}, \\
\tau_{k}=\text { const }, \quad 0<\tau_{k}<\tau_{\max }, \quad C_{k}^{(\tau)}=\mathrm{const} \in \mathbb{R}^{\left(N_{z c}, N_{z c}\right)}, \quad k=\overline{1, N_{\tau}} .
\end{gathered}
$$

Accordingly, equation (10) takes the form

$$
\begin{gathered}
\dot{\mathbf{z}}_{c}=B \mathbf{x}+C \mathbf{z}_{c}+\sum_{k=1}^{N_{\tau}} C_{k}^{(\tau)} \mathbf{z}_{c}\left(t-\tau_{k}\right)+\mathbf{f}_{1}\left(t, \mathbf{x}, \mathbf{z}_{c}\right), \\
\mathbf{f}_{1}\left(t, \mathbf{x}, \mathbf{z}_{c}\right)=\sum_{j=1,2,3, \ldots}\left(\left.\mathbf{z}_{c}\right|_{t_{j}+0}-\left.\mathbf{z}_{c}\right|_{t_{j}-0}\right) \delta\left(t-t_{j}\right)+ \begin{cases}\mathbf{f}_{c}^{(1)}\left(t, \mathbf{x}, s_{0}, \mathbf{z}_{c}\right), & t \in\left[0, t_{1}\right], \\
\mathbf{f}_{c}^{(1)}\left(t, \mathbf{x}, s_{k}, \mathbf{z}_{c}\right), & t \in\left[t_{k}, t_{k+1}\right],\end{cases} \\
k=1,2,3, \ldots
\end{gathered}
$$

In (15) $\left.\mathbf{z}_{c}\right|_{t_{j}+0}$ and $\left.\mathbf{z}_{c}\right|_{t_{j}-0}, j=1,2,3, \ldots$ are connected by the equation (6). 


\section{CONTROL OBJECTS WITH DISTRIBUTED PARAMETERS}

A dynamic system can contain not only a discrete controlling subsystem and control objects with lumped parameters, but also control objects with distributed parameters, dynamically connected with them across the boundaries. It is a hybrid dynamical system (HDS) [9], and its model equations contain a system of ordinary differential equations and partial differential equations with corresponding initial conditions connected by means of boundary conditions and constraint's conditions. In many cases that are important for applications from the point of view of the automatic control theory, objects with distributed parameters are linear and stationary. In this case, the considered non-linear HDS of a special type correspond to equations (1)-(5), (7)-(9), (15) and equations

$$
\begin{gathered}
\dot{\mathbf{z}}_{c}=B \mathbf{x}+C \mathbf{z}_{c}+A \mathbf{h}+\sum_{k=1}^{N_{\tau}} C_{k}^{(\tau)} \mathbf{z}_{c}\left(t-\tau_{k}\right)+\mathbf{f}_{1}\left(t, \mathbf{x}, \mathbf{z}_{c}\right), \quad \mathbf{h}=\int_{S} \mathbb{L}^{(H)} \mathbf{u} d S, \\
\partial \mathbf{u} / \partial t=\mathbb{L}_{1}^{(F)} \mathbf{u}+L_{2}^{(F)} \mathbf{x}+L_{3}^{(F)} \mathbf{z}_{c}+L_{4}^{(F)} \dot{\mathbf{z}}_{c}+L_{5}^{(F)} \mathbf{f}_{2}\left(t, \mathbf{x}, \mathbf{z}_{c}\right), \quad \mathbf{r} \in \Omega, \\
\left.\left(\mathbb{L}_{1}^{(G)} \mathbf{u}+L_{2}^{(G)} \mathbf{x}+L_{3}^{(G)} \mathbf{z}_{c}+L_{4}^{(F)} \mathbf{f}_{3}\left(t, \mathbf{x}, \mathbf{z}_{c}\right)\right)\right|_{S}=0, \quad S=\partial \Omega, \\
\mathbf{z}_{c}(t)=\mathbf{z}_{c_{0}}, \quad t \in\left[-\tau_{\max }, 0\right] ; \quad \mathbf{u}(\mathbf{r}, 0)=\mathbf{u}_{0}(\mathbf{r}) .
\end{gathered}
$$

Here $\mathbf{h}(t), \mathbf{h}: \mathbb{R} \rightarrow \mathbb{R}^{N_{h}}$ is the function characterizing the constraint's conditions; $A=$ const $\in \mathbb{R}^{\left(N_{y}, N_{h}\right)}$,

$$
\mathbf{f}_{2,3}\left(t, \mathbf{x}, \mathbf{z}_{c}\right)=\left\{\begin{array}{ll}
\mathbf{f}_{c}^{(2,3)}\left(t, \mathbf{x}, s_{0}, \mathbf{z}_{c}\right), & t \in\left[0, t_{1}\right], \\
\mathbf{f}_{c}^{(2,3)}\left(t, \mathbf{x}, s_{j}, \mathbf{z}_{c}\right), & t \in\left[t_{j}, t_{j+1}\right],
\end{array} \quad j=1,2,3, \ldots\right.
$$

$\mathbf{f}_{c}^{(2)}: \mathbb{R} \times \mathbb{R}^{N_{x}} \times S \times \mathbb{R}^{N_{z c}} \rightarrow \mathbb{R}^{N_{2}}, \mathbf{f}_{c}^{(3)}: \mathbb{R} \times \mathbb{R}^{N_{x}} \times S \times \mathbb{R}^{N_{z c}} \rightarrow \mathbb{R}^{N_{3}}$ are nonlinear functions that simulate the effects of switching in the discrete subsystem; $\mathbf{r} \in \Omega \subset \mathbb{R}^{N_{r}}$ are independent spatial coordinates of individual points of control objects with distributed parameters and their area; $L_{2}^{(F)}=L_{2}^{(F)}(\mathbf{r}), L_{2}^{(F)}: \mathbb{R}^{N_{r}} \rightarrow \mathbb{R}^{\left(N_{z c}, N_{x}\right)}, L_{3,4}^{(F)}=L_{3,4}^{(F)}(\mathbf{r})$, $L_{3,4}^{(F)}: \mathbb{R}^{N_{r}} \rightarrow \mathbb{R}^{\left(N_{z c}, N_{z c}\right)}, L_{5}^{(F)}=L_{5}^{(F)}(\mathbf{r}), L_{5}^{(F)}: \mathbb{R}^{N_{r}} \rightarrow \mathbb{R}^{\left(N_{z c}, N_{2}\right)}, L_{2}^{(G)}=L_{2}^{(G)}(\mathbf{r})$, $L_{2}^{(G)}: \mathbb{R}^{N_{r}} \rightarrow \mathbb{R}^{\left(N_{G}, N_{x}\right)}, L_{3}^{(G)}=L_{3}^{(G)}(\mathbf{r}), L_{3}^{(G)}: \mathbb{R}^{N_{r}} \rightarrow \mathbb{R}^{\left(N_{G}, N_{z c}\right)}, L_{4}^{(G)}=L_{4}^{(G)}(\mathbf{r})$, $L_{4}^{(G)}: \mathbb{R}^{N_{r}} \rightarrow \mathbb{R}^{\left(N_{G}, N_{3}\right)}$ are matrices that do not depend on time $t$ (similar to [10]); $\mathbb{L}_{1}^{(F)}:\left(\mathbb{R}^{N_{r}} \rightarrow \mathbb{R}^{N_{u}}\right) \rightarrow\left(\mathbb{R}^{N_{r}} \rightarrow \mathbb{R}^{N_{u}}\right), \mathbb{L}_{1}^{(H)}:\left(\mathbb{R}^{N_{r}} \rightarrow \mathbb{R}^{N_{u}}\right) \rightarrow\left(\mathbb{R}^{N_{r}} \rightarrow \mathbb{R}^{N_{h}}\right)$ are linear unbounded operators which do not depend on time $t ; \mathbb{L}_{1}^{(G)}:\left(\mathbb{R}^{N_{r}} \rightarrow \mathbb{R}^{N_{u}}\right) \rightarrow\left(\mathbb{R}^{N_{r}} \rightarrow \mathbb{R}^{N_{G}}\right)$ is a linear bounded or unbounded operator that does not depend on time $t ; \mathbf{u}=\mathbf{u}(\mathbf{r}, t)$, $\mathbf{u}: \mathbb{R}^{N_{r}} \times \mathbb{R} \rightarrow \mathbb{R}^{N_{u}}$. We assume that the initial conditions (17) correspond to the equilibrium state, i.e.

$$
\begin{gathered}
\mathbf{x}(t)=\mathbf{x}_{0}=\text { const, } \quad t<0, \\
\mathbf{f}_{k}^{(c)}\left(t, \mathbf{x}_{0}, s_{0}, \mathbf{z}_{c_{0}}\right)=\mathbf{f}_{k}^{(c)}\left(0, \mathbf{x}_{0}, s_{0}, \mathbf{z}_{c_{0}}\right), \quad t \leqslant 0, \quad k=1,2,3 \\
B \mathbf{x}_{0}+\left(C+\sum_{k=1}^{N_{\tau}} C_{k}^{(\tau)}\right) \mathbf{z}_{c_{0}}+A \mathbf{h}_{0}+\mathbf{f}_{1}^{(c)}\left(0, \mathbf{x}_{0}, s_{0}, \mathbf{z}_{c_{0}}\right)=0, \quad \mathbf{h}_{0}=\int_{S} \mathbb{L}^{(H)} \mathbf{u}_{0} d S \\
\mathbb{L}_{1}^{(F)} \mathbf{u}_{0}+L_{2}^{(F)} \mathbf{x}_{0}+L_{3}^{(F)} \mathbf{z}_{c_{0}}+L_{5}^{(F)} \mathbf{f}_{2}^{(c)}\left(0, \mathbf{x}_{0}, s_{0}, \mathbf{z}_{c_{0}}\right)=0, \quad \mathbf{r} \in \Omega \\
\left.\left(\mathbb{L}_{1}^{(G)} \mathbf{u}_{0}+L_{2}^{(G)} \mathbf{x}_{0}+L_{3}^{(G)} \mathbf{z}_{c_{0}}+L_{4}^{(F)} \mathbf{f}_{3}^{(c)}\left(0, \mathbf{x}_{0}, s_{0}, \mathbf{z}_{c_{0}}\right)\right)\right|_{S}=0, \quad S=\partial \Omega
\end{gathered}
$$




\section{LINEARIZED SYSTEM TRANSFER FUNCTIONS}

Consider (16) and (17) under the assumption $\mathbf{z}_{c_{0}}=0, \mathbf{u}_{0}(\mathbf{r})=0$.

The one-sided direct and inverse integral Laplace transform in time $t$ is

$$
\begin{gathered}
\tilde{f}(\lambda)=\mathscr{L}[f(t)]=\int_{0}^{\infty} f(t) e^{-\lambda t} d t, \quad f(t)=\mathscr{L}^{-1}[\tilde{f}(\lambda)]=\frac{1}{2 \pi i} \int_{\sigma-i \infty}^{\sigma+i \infty} \tilde{f}(\lambda) e^{\lambda t} d \lambda, \\
\sigma \geqslant \sigma_{a}, f: \mathbb{R} \rightarrow \mathbb{R}, \quad \tilde{f}: \mathbb{C} \rightarrow \mathbb{C}, \quad i=\sqrt{-1} .
\end{gathered}
$$

Here $\sigma_{a}$ is the abscissa of absolute convergence of the Laplace integral. Denote formally

$$
\tilde{\mathbf{f}}_{k}(\lambda)=\mathscr{L}\left[\mathbf{f}_{k}\left(t, \mathbf{x}(t), \mathbf{z}_{c}(t)\right)\right], \quad k=1,2,3 .
$$

Under homogeneous initial conditions $\mathbf{z}_{c_{0}}=0, \mathbf{u}_{0}(\mathbf{r})=0$ the equations (16), (17) after Laplace transform take the form

$$
\begin{gathered}
\lambda \tilde{\mathbf{z}}_{c}=B \tilde{\mathbf{x}}+\left(C+\sum_{k=1}^{N_{\tau}} C_{k}^{(\tau)} e^{-\tau_{k} \lambda}\right) \tilde{\mathbf{z}}_{c}+A \tilde{\mathbf{h}}+\tilde{\mathbf{f}}_{1} \\
\lambda \tilde{\mathbf{u}}=\mathbb{L}_{1}^{(F)} \tilde{\mathbf{u}}+L_{2}^{(F)} \tilde{\mathbf{x}}+\left(L_{3}^{(F)}+\lambda L_{4}^{(F)}\right) \tilde{\mathbf{z}}_{c}+L_{5}^{(F)} \tilde{\mathbf{f}}_{2}, \quad \mathbf{r} \in \Omega \\
\left.\left(\mathbb{L}_{1}^{(G)} \tilde{\mathbf{u}}+L_{2}^{(G)} \tilde{\mathbf{x}}+L_{3}^{(G)} \tilde{\mathbf{z}}_{c}+L_{4}^{(F)} \tilde{\mathbf{f}}_{3}\right)\right|_{S}=0, \quad S=\partial \Omega ; \quad \tilde{\mathbf{h}}=\int_{S} \mathbb{L}^{(H)} \tilde{\mathbf{u}} d S .
\end{gathered}
$$

From linearity (23) follows

$$
\tilde{\mathbf{h}}=B_{u}(\lambda) \tilde{\mathbf{x}}+C_{u}(\lambda) \tilde{\mathbf{z}}_{c}+C_{2}(\lambda) \tilde{\mathbf{f}}_{2}+C_{3}(\lambda) \tilde{\mathbf{f}}_{3}, \quad B_{u}(\bar{\lambda})=\overline{B_{u}(\lambda)}, \quad C_{u, 2,3}(\bar{\lambda})=\overline{C_{u, 2,3}(\lambda)} .
$$

Let be $\mathbf{e}_{1}^{(N)}=(1,0,0, \ldots, 0)^{T}, \mathbf{e}_{2}^{(N)}=(0,1,0, \ldots, 0)^{T}, \ldots, \mathbf{e}_{N}^{(N)}=(0,0,0, \ldots, 1)^{T}$.

From linearity (23), similarly to [10], linear boundary-value problems follow for finding separate columns of matrices $B_{u}(\lambda), C_{u}(\lambda), C_{2}(\lambda), C_{3}(\lambda)$ with fixed values $\lambda$

$$
\begin{gathered}
\lambda \mathbf{v}=\mathbb{L}_{1}^{(F)} \mathbf{v}+L_{2}^{(F)} \mathbf{e}_{j}^{\left(N_{x}\right)}, \quad \mathbf{r} \in \Omega,\left.\quad\left(\mathbb{L}_{1}^{(G)} \mathbf{v}+L_{2}^{(G)} \mathbf{e}_{j}^{\left(N_{x}\right)}\right)\right|_{S}=0 \\
B_{u}(\lambda) \mathbf{e}_{j}^{\left(N_{x}\right)}=\int_{S} \mathbb{L}^{(H)} \mathbf{v} d S, \quad j=\overline{1, N_{x}}, \\
\lambda \mathbf{v}=\mathbb{L}_{1}^{(F)} \mathbf{v}+\left(L_{3}^{(F)}+\lambda L_{3}^{(F)}\right) \mathbf{e}_{j}^{\left(N_{z c}\right)}, \quad \mathbf{r} \in \Omega, \\
\left.\left(\mathbb{L}_{1}^{(G)} \mathbf{v}+L_{3}^{(G)} \mathbf{e}_{j}^{\left(N_{z c}\right)}\right)\right|_{S}=0, \quad C_{u}(\lambda) \mathbf{e}_{j}^{\left(N_{z c}\right)}=\int_{S} \mathbb{L}^{(H)} \mathbf{v} d S, \quad j=\overline{1, N_{z c}} \\
\lambda \mathbf{v}=\mathbb{L}_{1}^{(F)} \mathbf{v}+L_{5}^{(F)} \mathbf{e}_{j}^{\left(N_{2}\right)}, \quad \mathbf{r} \in \Omega, \\
\left.\left(\mathbb{L}_{1}^{(G)} \mathbf{v}\right)\right|_{S}=0, \quad C_{2}(\lambda) \mathbf{e}_{j}^{\left(N_{2}\right)}=\int_{S} \mathbb{L}^{(H)} \mathbf{v} d S, \quad j=\overline{1, N_{2}}, \\
\lambda \mathbf{v}=\mathbb{L}_{1}^{(F)} \mathbf{v}, \quad \mathbf{r} \in \Omega, \\
\left.\left(\mathbb{L}_{1}^{(G)} \mathbf{v}+L_{4}^{(G)} \mathbf{e}_{j}^{\left(N_{3}\right)}\right)\right|_{S}=0, \quad C_{3}(\lambda) \mathbf{e}_{j}^{\left(N_{3}\right)}=\int_{S} \mathbb{L}^{(H)} \mathbf{v} d S, \quad j=\overline{1, N_{3}} .
\end{gathered}
$$

For small and medium values of $\lambda$ linear boundary value problems (25) can be solved numerically. When $|\lambda| \gg 1$ they are asymptotic integration. Substituting (24) into (22) leads to the result

$$
\left[\lambda E-C-\sum_{k=1}^{N_{\tau}} C_{k}^{(\tau)} e^{-\tau_{k} \lambda}-A C_{u}(\lambda)\right] \tilde{\mathbf{z}}_{c}=\left(B+A B_{u}(\lambda)\right) \tilde{\mathbf{x}}+
$$




$$
+\tilde{\mathbf{f}}_{1}+A C_{2}(\lambda) \tilde{\mathbf{f}}_{2}+A C_{3}(\lambda) \tilde{\mathbf{f}}_{3}, \quad E=\operatorname{diag}(1,1, \ldots, 1),
$$

where it comes from

$$
\tilde{\mathbf{z}}_{c}=\Phi(\lambda) \tilde{\mathbf{x}}+\sum_{k=1}^{3} \Phi_{k}(\lambda) \tilde{\mathbf{f}}_{k}
$$

In $(27)$ the transfer function matrices are represented as quasi-rational fractions $[9,10]$

$$
\begin{gathered}
\Phi_{1}(\lambda)=\left[\lambda E-C-\sum_{k=1}^{N_{\tau}} C_{k}^{(\tau)} e^{-\tau_{k} \lambda}-A C_{u}(\lambda)\right]^{-1}=Q_{1}(\lambda) / D(\lambda), \\
\Phi(\lambda)=\Phi_{1}(\lambda)\left(B+A B_{u}(\lambda)\right)=Q(\lambda) / D(\lambda), \\
\Phi_{2}(\lambda)=\Phi_{1}(\lambda) A C_{2}(\lambda)=Q_{2}(\lambda) / D(\lambda), \quad \Phi_{3}(\lambda)=\Phi_{1}(\lambda) A C_{3}(\lambda)=Q_{3}(\lambda) / D(\lambda), \\
D(\lambda)=\operatorname{det}\left[\lambda E-C-\sum_{k=1}^{N_{\tau}} C_{k}^{(\tau)} e^{-\tau_{k} \lambda}-A C_{u}(\lambda)\right] .
\end{gathered}
$$

Here $D: \mathbb{C} \rightarrow \mathbb{C}$ is the characteristic quasi-polynomial of HDS, $Q: \mathbb{C} \rightarrow \mathbb{C}^{\left(N_{z c}, N_{x}\right)}$, $Q_{1}: \mathbb{C} \rightarrow \mathbb{C}^{\left(N_{z c}, N_{z c}\right)}, Q_{2}: \mathbb{C} \rightarrow \mathbb{C}^{\left(N_{z c}, N_{2}\right)}, Q_{3}: \mathbb{C} \rightarrow \mathbb{C}^{\left(N_{z c}, N_{3}\right)}$ are matrices whose elements are perturbing quasi-polynomials of HDS. In [10] the conditions for the analyticity of the functions $B_{u}(\lambda)$ and $C_{u}(\lambda)$, as well as the characteristic and perturbing quasi-polynomials $D(\lambda)$ and $Q(\lambda)$ are formulated and proved in the domain $|\lambda| \gg 1$, $\operatorname{Re} \lambda>-|\lambda| \sin \alpha, 0<\alpha<\pi / 2$ (conditions with respect to the functions $C_{2}(\lambda)$ and $C_{3}(\lambda)$, as well as perturbing quasi-polynomials $Q_{1}(\lambda), Q_{2}(\lambda)$ and $Q_{3}(\lambda)$ can be formulated and proved similarly). In particular, with sufficiently general assumptions, this is true for a number of typical mathematical models of control objects with distributed parameters (heat conduction and diffusion processes, damping and supporting layers of a viscous incompressible fluid, the dynamics of an elastic medium with a small but finite energy dissipation).

As a rule, for the most common mathematical models of control objects with distributed parameters

$$
\begin{gathered}
\left\|B_{u}(\lambda)\right\|={ }^{\circ}(|\lambda|), \quad\left\|C_{u}(\lambda)\right\|={ }^{\circ}(|\lambda|), \quad\left\|C_{2}(\lambda)\right\|={ }^{\circ}(|\lambda|), \quad\left\|C_{3}(\lambda)\right\|={ }^{\circ}(|\lambda|), \\
\lambda \rightarrow \infty, \operatorname{Re} \lambda>-|\lambda| \sin \alpha, \quad 0<\alpha<\pi / 2 .
\end{gathered}
$$

\section{HYBRID AUTOMATA MODEL EXTENSION}

Definition 1. By the extended model of a hybrid automaton, we mean a mathematical model in which the switching dynamics of a discrete subsystem is modeled by equations (1)-(5), (7), (12), and the dynamics of a continuous subsystem is modeled by some integral or integro-differential equations.

Lemma 1. If the functions $B_{u}(\lambda), C_{u}(\lambda), C_{2}(\lambda), C_{3}(\lambda)$ are analytic with $|\lambda| \gg 1$, $\operatorname{Re} \lambda>-|\lambda| \sin \alpha, 0<\alpha<\pi / 2$, and conditions (29) are satisfied, then under homogeneous initial conditions $\mathbf{z}_{c_{0}}=0, \mathbf{u}_{0}(\mathbf{r})=0$ the nonlinear HDS (16), (17) reduces to a nonlinear integral convolution type equation.

Proof. In this case

$$
\Phi(\lambda) \rightarrow 0, \quad \Phi_{k}(\lambda) \rightarrow 0, \quad k=1,2,3, \quad \lambda \rightarrow \infty, \operatorname{Re} \lambda>-|\lambda| \sin \alpha, \quad 0<\alpha<\pi / 2
$$


and transfer functions are analytical in domain (30). In this case, for $t \neq 0$ and sufficiently large $\sigma$ the Mellin integral (20) for inversion of the Laplace transform converges, and there exist matrices of impulse transition functions

$$
\begin{gathered}
F(t)=\mathscr{L}^{-1}[\Phi(\lambda)], \quad F_{k}(t)=\mathscr{L}^{-1}\left[\Phi_{k}(\lambda)\right], \\
\|F(t)\|<\infty, \quad\left\|F_{k}(t)\right\|<\infty, \quad 0<t<\infty \\
F(t)=F_{k}(t) \equiv 0, \quad t<0, \quad k=1,2,3
\end{gathered}
$$

wherein

$$
\begin{gathered}
\lim _{t \rightarrow+0} F_{1}(t)=\lim _{\lambda \rightarrow \infty} \lambda \Phi_{1}(\lambda)=E, \quad \lim _{t \rightarrow+0} \int_{0}^{t} F(\xi) d \xi=\lim _{\lambda \rightarrow \infty} \lambda \Phi(\lambda)=0, \\
\lim _{t \rightarrow+0} \int_{0}^{t} F_{k}(\xi) d \xi=\lim _{\lambda \rightarrow \infty} \lambda \Phi_{k}(\lambda)=0, \quad k=2,3 .
\end{gathered}
$$

Reversing the integral Laplace transform in (27), we find

$$
\mathbf{z}_{c}(t)=\int_{0}^{t}\left[F(t-\xi) \mathbf{x}(\xi)+\sum_{k=1}^{3} F_{k}(t-\xi) \mathbf{f}_{k}\left(\xi, \mathbf{x}(\xi), \mathbf{z}_{c}(\xi)\right)\right] d \xi
$$

Equation (33) is a non-linear integral equation of convolution type for the unknown function $\mathbf{z}_{c}(t)$.

When $0<t \ll 1$ and when $t \gg 1$ for the inversion of the integral Laplace transform in (31) approximate formulas can be obtained on the basis of its asymptotic properties. For medium values of $t$ the inversion of the Laplace transform in (31) can be performed numerically.

Lemma 2. If the initial conditions (17) correspond to the equilibrium state, i.e. conditions (19) are fulfilled, functions $B_{u}(\lambda), C_{u}(\lambda), C_{2}(\lambda), C_{3}(\lambda)$ are analytic with $|\lambda| \gg 1, \operatorname{Re} \lambda>-|\lambda| \sin \alpha, 0<\alpha<\pi / 2$, and conditions (29) are satisfied, then nonlinear HDS (16), (17) reduces to a nonlinear integral convolution type equation.

Proof. Putting $\mathbf{x}^{*}(t)=\mathbf{x}(t)-\mathbf{x}_{0}, \mathbf{z}_{c}^{*}(t)=\mathbf{z}_{c}(t)-\mathbf{z}_{c_{0}}, \mathbf{u}^{*}(\mathbf{r}, t)=\mathbf{u}(\mathbf{r}, t)-\mathbf{u}_{0}(\mathbf{r}), \mathbf{h}^{*}(t)=$ $=\mathbf{h}(t)-\mathbf{h}_{0}, \mathbf{f}_{k}^{*}\left(t, \mathbf{x}^{*}, \mathbf{z}_{c}^{*}\right)=\mathbf{f}_{k}\left(t, \mathbf{x}_{0}+\mathbf{x}^{*}(t), \mathbf{z}_{c_{0}}+\mathbf{z}_{c}^{*}(t)\right)-\mathbf{f}_{k}\left(0, \mathbf{x}_{0}, \mathbf{z}_{c_{0}}\right)$, we obtain a system of equations for quantities ()$^{*}$, similar to (16) with homogeneous initial conditions, whence it follows $\mathbf{z}_{c}^{*}(t)=\int_{0}^{t}\left[F(t-\xi) \mathbf{x}^{*}(\xi)+\sum_{k=1}^{3} F_{k}(t-\xi) \mathbf{f}_{k}^{*}\left(\xi, \mathbf{x}^{*}(\xi), \mathbf{z}_{c}^{*}(\xi)\right)\right] d \xi$. We get

$$
\begin{gathered}
\mathbf{z}_{c}(t)=\mathbf{z}_{c_{0}}+\int_{0}^{t}\left[F(t-\xi)\left(\mathbf{x}(\xi)-\mathbf{x}_{0}\right)+\right. \\
\left.+\sum_{k=1}^{3} F_{k}(t-\xi)\left[\mathbf{f}_{k}\left(\xi, \mathbf{x}(\xi), \mathbf{z}_{c}(\xi)\right)-\mathbf{f}_{k}\left(0, \mathbf{x}_{0}, \mathbf{z}_{c_{0}}\right)\right]\right] d \xi
\end{gathered}
$$

after returning to the original variables.

Theorem 1. If the initial conditions (17) correspond to the equilibrium state, i.e. conditions (19) are fulfilled, functions $B_{u}(\lambda), C_{u}(\lambda), C_{2}(\lambda), C_{3}(\lambda)$ are analytic with $|\lambda| \gg 1, \operatorname{Re} \lambda>-|\lambda| \sin \alpha, 0<\alpha<\pi / 2$, and conditions (29) are fulfilled, then nonlinear special type HDS is isomorphic to an extended model of a hybrid automaton in which the dynamics of a continuous subsystem is modeled by a nonlinear integral convolution type equation. 
Proof. On the basis of Lemmas 1 and 2 and using (15) and (33), we find

$$
\begin{gathered}
\mathbf{z}_{c}(t)=\mathbf{z}_{c_{0}}+\int_{0}^{t}\left[F(t-\xi)\left(\mathbf{x}(\xi)-\mathbf{x}_{0}\right)+\right. \\
\left.+\sum_{k=1}^{3} F_{k}(t-\xi)\left[\mathbf{f}_{c}^{(k)}\left(\xi, \mathbf{x}(\xi), s_{d_{0}}, \mathbf{z}_{c}(\xi)\right)-\mathbf{f}_{c}^{(k)}\left(\xi, \mathbf{x}_{0}, s_{d_{0}}, \mathbf{z}_{c_{0}}\right)\right]\right] d \xi, \quad t \in\left[0, t_{1}\right] \\
\mathbf{z}_{c}(t)=\mathbf{z}_{c_{0}}+\sum_{j=1}^{m}\left(\left.\mathbf{z}_{c}\right|_{t_{j}+0}-\left.\mathbf{z}_{c}\right|_{t_{j}-0}\right) F_{1}\left(t-t_{j}\right)+\int_{0}^{t}\left[F(t-\xi)\left(\mathbf{x}(\xi)-\mathbf{x}_{0}\right)+\right. \\
\left.+\sum_{k=1}^{3} F_{k}(t-\xi)\left[\mathbf{f}_{c}^{(k)}\left(\xi, \mathbf{x}(\xi), s_{d_{m}}, \mathbf{z}_{c}(\xi)\right)-\mathbf{f}_{c}^{(k)}\left(0, \mathbf{x}_{0}, s_{d_{0}}, \mathbf{z}_{c_{0}}\right)\right]\right] d \xi \\
t \in\left[t_{m}, t_{m+1}\right], \quad m=1,2,3, \ldots, \quad t_{m} \in T
\end{gathered}
$$

The extended model of the hybrid automaton corresponds to equations (1)-(5), (7), (12) and (35), where $\left.\mathbf{z}_{c}\right|_{t_{j}+0}$ and $\left.\mathbf{z}_{c}\right|_{t_{j}-0}$ are related by equation (5).

The generalized degree $n \in \mathbb{R}$ of the characteristic quasi-polynomial $D(\lambda)$ is determined from the condition $[9,10]$

$$
\lim _{\lambda \rightarrow \infty} \lambda^{-n} D(\lambda)=c_{a}, 0<\left|c_{a}\right|<\infty, \quad \lambda \rightarrow \infty, \quad \operatorname{Re} \lambda>\sigma_{0}, \quad \sigma_{0} \in(-\infty, 0)
$$

When conditions (29) are fulfilled, $n=N_{z c}$, and condition (36) is fulfilled in a wider domain $\lambda \rightarrow \infty$, Re $\lambda>-|\lambda| \sin \alpha, 0<\alpha<\pi / 2$.

The numerical solution of auxiliary linear boundary value problems (25) with $|\lambda|<R$, $R \gg 1, \operatorname{Re} \lambda>-\varepsilon, 0<\varepsilon \ll 1$ is performed on the basis of the Galerkin projection method [10]. In the considered domain, the absence of singularities of functions $B_{u}(\lambda), C_{u}(\lambda), C_{2}(\lambda), C_{3}(\lambda)$ and, consequently, of the characteristic and perturbing quasipolynomials $D(\lambda), Q(\lambda), Q_{1}(\lambda), Q_{2}(\lambda), Q_{3}(\lambda)$ is verified numerically on the basis of principle of argument. The presence of singularities of the characteristic and perturbing quasi-polynomials when $\operatorname{Re} \lambda>0$ means the instability of control objects with distributed parameters as such and requires a change in the parameters of the designed system. Further, we assume that the functions $B_{u}(\lambda), C_{u}(\lambda), C_{2}(\lambda), C_{3}(\lambda)$, and also $D(\lambda), Q(\lambda)$, $Q_{1}(\lambda), Q_{2}(\lambda), Q_{3}(\lambda)$ are analytic in domain $\operatorname{Re} \lambda>\sigma_{0}, \sigma_{0} \in(-\infty, 0)$.

If the quasi-polynomial $D(\lambda)$ is analytic with $\operatorname{Re} \lambda>\sigma_{0}, \sigma_{0} \in(-\infty, 0)$ and is stable, that is, all its roots (zeros) lie strictly to the left of the imaginary axis $\operatorname{Re} \lambda=0$, then according to the theorem on the stable quasi-polynomial [9]

$$
\underset{0 \leqslant \omega \leqslant \infty}{\Delta} \arg D(i \omega)=\frac{1}{2} n \pi
$$

When optimizing the frequency change step $\omega$ (37) this is a "fast" algorithm for checking the stability of linearized HDS [9].

Theorem 2. If the initial conditions (17) correspond to the equilibrium state, the functions $B_{u}(\lambda), C_{u}(\lambda), C_{2}(\lambda), C_{3}(\lambda)$ are analytic with $|\lambda| \gg 1, \operatorname{Re} \lambda>-|\lambda| \sin \alpha$, $0<\alpha<\pi / 2$, conditions (29) are satisfied, and the characteristic quasi-polynomial $D(\lambda)$ is stable, then the kernel of nonlinear integral equations of convolution type (35) decays exponentially.

Proof. By the conditions of the theorem, the characteristic quasi-polynomial $D(\lambda)$ is analytic in domain $\operatorname{Re} \lambda>\sigma_{0}, \sigma_{0} \in(-\infty, 0)$. 


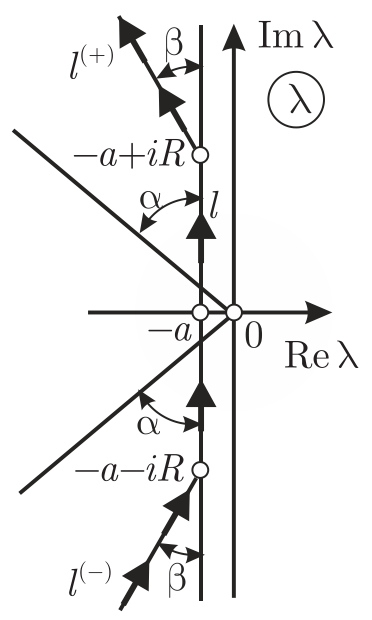

Fig. 2. Integration contour

All the roots (zeros) of $D(\lambda)$ lie strictly to the left of the imaginary axis $\operatorname{Re} \lambda=0$. Consequently, all the singular points of the transfer functions (28) lie strictly to the left of the imaginary axis $\operatorname{Re} \lambda=0$. If conditions (29) are true, then from (30) it follows that the singular points of the transfer functions cannot be in the domain $\operatorname{Re} \lambda>-|\lambda| \sin \alpha, 0<\alpha<\pi / 2,|\lambda| \gg 1$. In formulas (31) the path of integration in the Mellin integral (20) for inversion of the Laplace transform can be along a straight line $\operatorname{Re} \lambda=-a, a>0$. Further, it can be deformed into the contour $l^{(-)} \cup l \cup l^{(+)}$(Fig. 2), completely lying in the domain where the transfer functions are analytical. In particular,

$$
\begin{gathered}
F(t)=\frac{1}{2 \pi i} \int_{l^{(-)} \cup l \cup l(+)} \Phi(\lambda) e^{\lambda t} d \lambda, \quad l=\{\lambda=-a+i \omega, \quad \omega \in[-R, R]\}, \\
l^{( \pm)}=\left\{\lambda=-a \pm i R \pm i e^{ \pm i \beta} \xi, \quad \xi \in[0, \infty)\right\}, \quad R \gg 1, \quad 0<\beta<\alpha<\pi / 2, \quad a>0, \\
\int_{l} \Phi(\lambda) e^{\lambda t} d \lambda=\frac{1}{t}\left[\left.\left(\Phi(\lambda) e^{\lambda t}\right)\right|_{\lambda=-a-i R} ^{\lambda=a-i R}-\int_{l} \Phi^{\prime}(\lambda) e^{\lambda t} d \lambda\right]={ }^{\circ}\left(e^{-a t}\right), \quad t \rightarrow \infty, \\
\left|\int_{l^{( \pm)}} \Phi(\lambda) e^{\lambda t} d \lambda\right| \leqslant M e^{-a t} \int_{0}^{\infty} e^{-\xi t \sin \beta} d \xi=\frac{M}{t \sin \beta} e^{-a t}={ }^{\circ}\left(e^{-a t}\right), \quad t \rightarrow \infty, \\
M=\max _{\xi \in[0, \infty)}\left|\Phi\left(-a \pm i R \pm i e^{ \pm i \beta} \xi\right)\right|<\infty .
\end{gathered}
$$

Those $F(t)={ }^{\bar{o}}\left(e^{-a t}\right), t \rightarrow \infty$, and similarly $F_{k}(t)={ }^{\bar{o}}\left(e^{-a t}\right), t \rightarrow \infty, k=1,2,3$, $a>0$.

Corollary 2. Under the conditions of Theorem 2, the asymptotic complexity of the numerical implementation of the extended model of a hybrid automaton based on integral equations (35) has the same order as the asymptotic complexity of the numerical implementation of the model of a hybrid automaton based on Cauchy problems for ordinary differential equations (6).

Remark 1. The numerical integration of linear boundary value problems (25) and the numerical inversion of the integral Laplace transform in (31) can be performed on a sufficiently powerful computing system. Further numerical solution of integral equations (35) can be performed on the embedded computer system.

\section{References}

1. Kashevnik A. M., Ponomarev A. V., Savosin S. V. Hybrid Systems Control Based on Smart Space Technology. SPIIRAS Proceedings, 2014, iss. 4(35), pp. 212-226 (in Russian).

2. Meslem N., Ramdani M., Candau Y. Guaranteed Parameter Set Estimation for Monotone Dynamical Systems Using Hybrid Automata. In: Reliable Computing, Springer Verlag, 2010, pp. 88-104.

3. Karoui M. F., Alla H., Chatti A. Monitoring of dynamic processes by rectangular hybrid automata. Nonlinear Analysis: Hybrid Systems, 2010, vol. 4, iss. 4, pp. 766-774. DOI: https://doi.org/10.1016/j.nahs.2010.05.004 
4. Thiagarajan P. S., Yang S. Modular discrete time representation of distributed hybrid automata. Theoretical Computer Science, 2012, vol. 429, pp. 292-304. DOI: https://doi.org/10.1016/j.tcs.2011.12.050

5. Konećný M., Taha W., Bartha F. A., Duracz J., Duracz A., Ames A. D. Enclosing the behavior of a hybrid automation up to and beyond a Zeno point. Nonlinear Analysis: Hybrid Systems, 2016, vol. 20, pp. 1-20. DOI: https://doi.org/10.1016/j.nahs.2015.10.004

6. Elmetennani S., Laleg-Kirati T.M., Djemai M., Tadjine M. New MPPT algorithm for PV applications based on hybrid dynamical approach. Journal of Process Control, 2016, vol. 48, pp. 14-24. DOI: https://doi.org/10.1016/j.jprocont.2016.10.001

7. Iovine A., Valentini F., De Santis E., Di Benedetto M. D., Pratesi M. Safe human-inspired mesoscopic hybrid automation for autonomous vehicles. Nonlinear Analysis: Hybrid Systems, 2017, vol. 25, pp. 192-210. DOI: https://doi.org/10.1016/j.nahs.2016.08.008

8. Shornikov Yu., Bessonov A., Dostovalov D. Specification and instrumental analysis of hybrid systems. Science Bulletin of the Novosibirsk State Technical University, 2015, no. 4(61), pp. 101-117 (in Russian). DOI: http://dx.doi.org/10.17212/1814-1196-2015-4101-117

9. Andreichenko D. K., Andreichenko K. P. On the theory of hybrid dynamical systems. Journal of Computer and Systems Sciences International, 2000, vol. 39, no. 3, pp. 383398.

10. Portenko M. S., Melnichuk D. V., Andreichenko D. K. Analyticity conditions of characteristic and disturbing quasipolynomials of hybrid dynamical systems. Izv. Saratov Univ. (N. S.), Ser. Math. Mech. Inform., 2016, vol. 16, no. 2, pp. 208-217 (in Russian). DOI: https://doi.org/10.18500/1816-9791-2016-16-2-208-217

\section{Cite this article as:}

Andreichenko D. K., Andreichenko K. P., Batraeva I. A. Hibrid Automation Extended Model. Izv. Saratov Univ. (N. S.), Ser. Math. Mech. Inform., 2019, vol. 19, iss. 1, pp. 94-104. DOI: https://doi.org/10.18500/1816-9791-2019-19-1-94-104

УДК $519.713 .8: 517.935 .2$

\section{Расширенная модель гибридного автомата}

\section{Д. К. Андрейченко, К. П. Андрейченко, И. А. Батраева}

Андрейченко Дмитрий Констатинович, доктор фризико-математических наук, заведующий касредрой математического обеспечения вычислительных комплексов и инсрормационных систем, Саратовский национальный исследовательский государственный университет имени Н. Г. Чернышевского, Россия, 410012, Саратов, ул. Астраханская, д. 83, andreichenkodk@gmail.com

Андрейченко Константин Петрович, доктор технических наук, просрессор кафредры прикладной математики и системного анализа, Саратовский государственный технический университет имени Гагарина Ю. А, Россия, 410054, Саратов, ул. Политихническая, д. 77, kp_andreichenko@ renet.ru

Батраева Инна Александровна, кандидат фризико-математических наук, и.о. заведующего кафредрой технологий программирования, Саратовский национальный исследовательский государственный университет имени Н. Г. Чернышевского, Россия, 410012, Саратов, ул. Астраханская, д. 83, batraevaia@info.sgu.ru 
Рассмотрена расширенная модель гибридных автоматов для динамических систем, где наряду с дискретной управляющей подсистемой и объектами управления с сосредоточенными по пространству параметрами имеются объекты управления с распределенными по пространству параметрами (линейные и стационарные с точки зрения теории автоматического управления). Показана возможность программной реализации расширенной модели гибридных автоматов на встроенных вычислительных системах.

Ключевые слова: гибридные автоматы, гибридные динамические системы, встроенные вычислительные системы.

Образец для цитирования:

Andreichenko D. K., Andreichenko K. P., Batraeva I. A. Hibrid Automation Extended Model [Андрейченко Д. К., Андрейченко К. П., Батраева И. А. Расширенная модель гибридного автомата] // Изв. Сарат. ун-та. Нов. сер. Сер. Математика. Механика. Информатика. 2019. T. 19, вып. 1. С. 94-104. DOI: https://doi.org/10.18500/1816-9791-2019-19-1-94-104 